\title{
Ophthalmic Dosage Form
}

National Cancer Institute

\section{Source}

National Cancer Institute. Ophthalmic Dosage Form. NCI Thesaurus. Code C69039.

A substance intended for administration in or around the eye. 